\title{
Le corps médical a-t-il absolument besoin de ses propres données?
}

Renato Laffranchi ${ }^{a}$, Urs Stoffel ${ }^{b}$

a General manager NewIndex

b Président du conseil d'administration de NewIndex, membre du Comité central de la FMH
Correspondance: Dr Renato Laffranchi

NewIndex SA

Leberngasse 19

CH-4600 Olten

renato.laffranchi[at]newindex.ch
Au fil des discussions, il n'est pas rare d'entendre: «A quelles fins les médecins suisses ont-ils besoin de leurs propres données et à quoi leur sert une telle collecte?» ou «N'avons-nous pas déjà suffisamment de chiffres de la part des caisses-maladie, de l'OFSP et de l'Office fédéral de la statistique (OFS)?»; «Recueillir ses propres données coûte beaucoup d'argent et n'apporte pas de véritables avantages.»

Dans ces colonnes, nous tentons de démontrer l'importance et la nécessité de telles données pour le corps médical suisse et de rappeler que celles-ci ne peuvent pas être simplement remplacées par celles des assureurs ou de l'OFS.

\section{Qui est NewIndex et que fait-elle?}

La société NewIndex SA a été fondée en 2000 en vue de répondre aux exigences tarifaires du TARMED. Elle appartient à 100\% aux sociétés cantonales de médecine, à la FMH et à la Caisse des médecins. NewIndex a pour mission de créer un pool regroupant les données de toute la Suisse, entre autres dans le but de ou pour des groupes de médecins en se fondant sur les données des médecins. NewIndex répond également à des mandats (analyse et évaluation) émis par des autorités sanitaires cantonales ou fédérales, des instituts scientifiques et autres institutions du domaine de la santé.

\section{La priorité: protection des données et protection contre les abus en la matière}

Une grande partie de l'activité de NewIndex repose sur la collecte des données et donc aussi sur la confiance que lui accordent les médecins fournissant volontairement leurs données pour le Centre national de consolidation des données, plus connu sous le nom de NAKO. Ces praticiens sont en droit d'attendre que NewIndex traite leurs données sensibles avec diligence, confidentialité et de manière conforme à la protection des données. Toute entreprise qui remettrait en cause ce «capital confiance» par un manque de soin dans la gestion des données serait en effet vouée à l'échec.

\section{Newlndex: créer un pool regroupant les données de toute la Suisse dans le but de compiler les informations pour mener les négociations tarifaires et offrir un instrument aux médecins dans le cadre de l'évaluation de l'économicité.}

compiler les informations du corps médical pour mener les négociations tarifaires avec les assureurs et d'offrir un instrument aux médecins dans le cadre de l'évaluation de l'économicité. Pour ce faire, dix centres de confiance (Trust Centers) ont vu le jour sur l'ensemble du territoire grâce au soutien de NewIndex. Les sociétés cantonales de médecine ont chacune conclu une convention avec le centre de confiance de leur canton, établissant les modalités de collecte des données.

En fournissant des évaluations, des analyses ou des ébauches de modèle, NewIndex apporte son concours aux deux associations professionnelles nationales, la CCM et la FMH, aussi bien lors des négociations sur la valeur du point tarifaire que durant la révision de la structure tarifaire du TARMED. En outre, NewIndex entreprend également des préparations de données et d'évaluations sur mesure à l'intention de diverses sociétés cantonales ou spécialisées,
C'est la raison pour laquelle deux instances veillent à une utilisation conforme et diligente des données: le «comité NAKO» et le code NAKO. Le premier se compose de représentants de la FMH, des sociétés cantonales de médecine, de diverses organisations faîtières, d'un représentant des centres de confiance, d'un délégué de NewIndex et d'un conseiller juridique; quant au second, il règlemente l'accès et l'exploitation des données par des associations et des tiers.

\section{Les centres de confiance: le noyau de la collecte nationale des données du corps médical suisse}

Les dix centres de confiance collectent de manière décentralisée les données de facturation des médecins, qui sont ensuite consolidées par le NAKO. Mais ces centres de confiance remplissent encore bien davantage de missions. Par leur proximité avec les méde- 


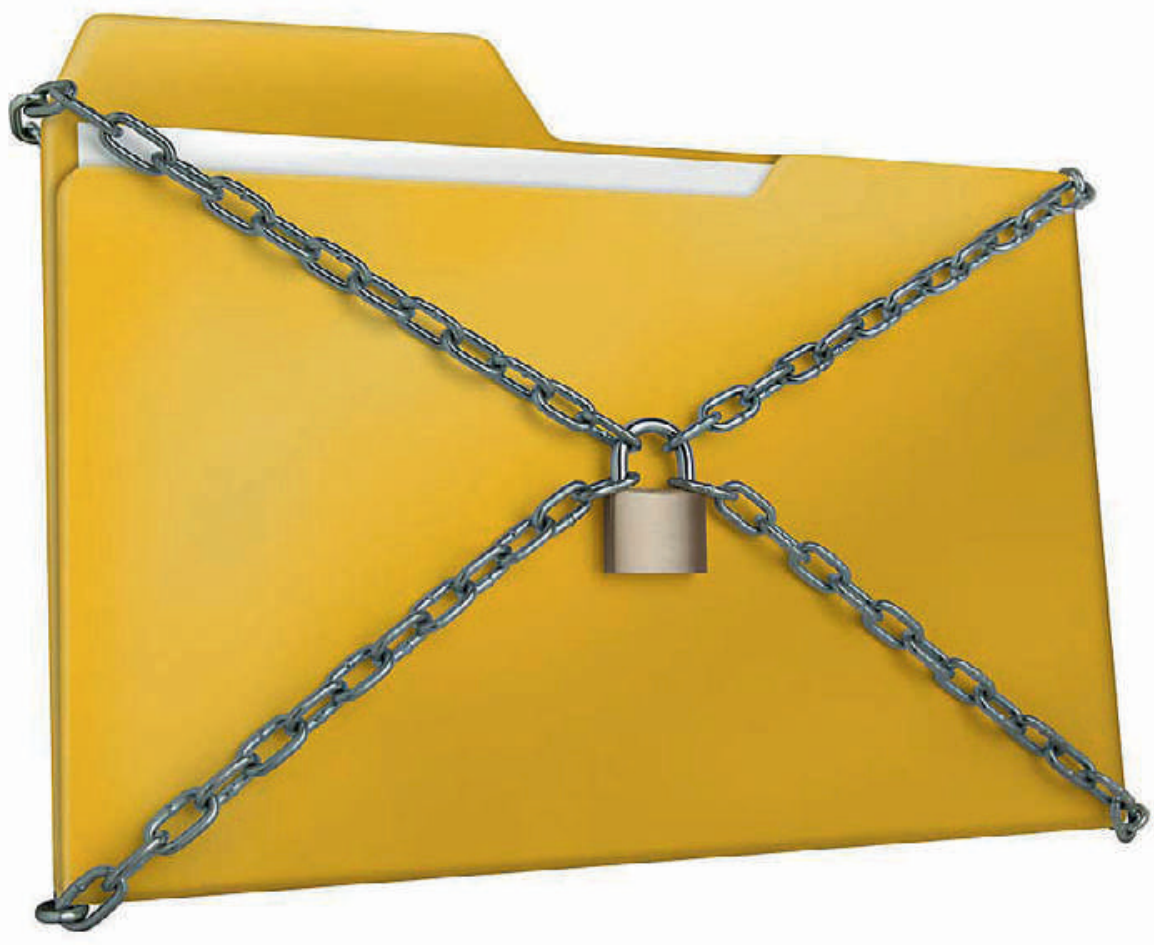

La priorité: protection des données et protection contre les abus en la matière.

cins et leur capacité à les fidéliser, ils établissent un climat de confiance avec les praticiens qui leur sont rattachés, ils les conseillent et les accompagnent dans tout ce qui concerne les aspects économiques de la gestion de leur cabinet. Grâce à l'aide de leur centre de confiance, de nombreux médecins ont pu éviter une demande de restitution injustifiée (procédures EAE) aux caisses-maladie. Les centres de confiance jouent ainsi un rôle important de protection pour les médecins indépendants et garantissent dans une large mesure la collecte de données au niveau national. Pour encadrer leurs droits et leurs devoirs respectifs, ils concluent des contrats de mandats avec les sociétés cantonales de médecine concernées.

Afin que la collecte puisse fonctionner au niveau national, il est indispensable que les données soient recueillies selon des standards uniformes et contraignants pour tous les centres de confiance. C'est seulement ainsi que les données pourront être consolidées par le NAKO, et leur compatibilité garantie. Par ailleurs, les centres de confiance doivent participer au financement nécessaire à la consolidation des données tant que celui-ci ne sera pas assumé par les sociétés cantonales de médecine.

\section{Newlndex: bureau technique de la Conférence des sociétés cantonales de médecine (CCM)}

NewIndex se charge de tout le bureau technique de la CCM. Dans le cadre des négociations avec tarifsuisse sur la CPP (LeiKoV), elle analyse et contrôle l'exactitude des données fournies par les assureurs et indique d'éventuelles irrégularités. Par ailleurs, elle les compare avec celles du corps médical compilées dans le pool de données national. Si ces données ne présentent pas une évolution similaire, elle recherche les explications possibles et met en évidence les différences. NewIndex élabore ses propres propositions de modèle dans le but d'établir un modèle de substitution pour la CPP. En 2013, et c'est nouveau, NewIndex a également participé aux négociations sur la valeur du point tarifaire avec la deuxième communauté d'achat (HSK).

\section{Newlndex: l'épine dorsale de la révision tarifaire du TARMED (TARVISION)}

Pour la FMH, NewIndex réalise également des évaluations et des analyses dans différents domaines, et notamment pour le projet Tarvision. Dans ce cas, une bonne représentativité des spécialités est indispensable à la réussite du projet. En effet, pour que les différentes sociétés de discipline puissent faire valoir leur point de vue, la plausibilité de leurs attentes doit être étayée par des chiffres et des données fiables.

\section{Newlndex: centre de référence des données de facturation, notamment en vue de défendre le point de vue des médecins} Grâce aux évaluations de NewIndex, des modèles pour de nouveaux tarifs de laboratoire ont vu le jour. Lors des négociations sur une rémunération sans marge de la propharmacie avec les assureurs, elles ont permis de montrer que la durée des consultations reste la même avec ou sans remise de médicaments.

Les sociétés cantonales de médecine et les sociétés de discipline bénéficient également du soutien de NewIndex. En 2012, cette dernière a notamment élaboré un argumentaire complet à l'intention de la société des médecins du canton de Schwytz, grâce auquel une augmentation de la valeur du point tarifaire de 2 centimes a pu être obtenue. En effet, suite à un blocage par les assureurs, seules les données NAKO des médecins étaient disponibles à cette époque. C'est grâce à elles qu'il a été possible de motiver une augmentation de la valeur du point tarifaire.

De la même manière, NewIndex a réalisé des analyses pour le canton d'Argovie et développé des modèles qui ont finalement abouti à une hausse de la valeur du point tarifaire fixée par le canton. En outre, NewIndex apporte son concours au canton d'Argovie lors de la campagne de votation sur l'autorisation de la propharmacie («Wahlfreiheit beim Medikamentenbezug»).

Par ailleurs, NewIndex a également contribué au développement du concept de la concentration thérapeutique, élaboré en collaboration avec la Société suisse d'oncologie médicale. Il s'est agi notamment de montrer que tous les oncologues ne traitent pas dans la même mesure des patients oncologiques, mais qu'ils exercent également dans des proportions différentes en médecine interne. 


\section{Un socle de données pour une étude sur les soins orientée vers l'avenir}

Avec l'aide d'OBELISC (Objective Evaluation and Leadership In Scientific Health Data Collection), NewIndex pénètre de plus en plus le domaine de la recherche sur les soins: sur mandat de la direction de la santé du canton de Schwytz, elle a ainsi mené un projet en collaboration avec le centre de confiance medkey visant à analyser et représenter la situation du canton en matière de soins. Les données OBELISC, un sous-collectif de données spécialement créé par NewIndex, permettent aujourd'hui de soutenir également des projets externes de recherche universitaire.

Grâce à cela, NewIndex s'élève au rang de «laboratoire d'idées» cherchant à répondre aux questions et à anticiper les enjeux de la prise en charge médicale de demain. Afin qu'une telle recherche sur les soins soit fiable pour l'avenir, elle doit pouvoir compter sur un collectif de données approprié et susceptible d'apporter des conclusions valides et pertinentes.

\section{La signification politique du pool de données}

Si le corps médical suisse entend ouvrir des perspectives et influencer de manière décisive l'avenir de la prise en charge et du système de santé, il doit disposer de ses propres données, pouvoir faire appel à des évaluations et à des analyses en vue de répondre aux questions en suspens, et surtout proposer des solutions acceptables pour l'avenir de la santé dans notre pays. Qui d'autre, si ce n'est le corps médical, est en mesure de façonner cet avenir et de le marquer de son empreinte? En créant le pool de données national du corps médical, nous ouvrons dans ce sens en étroite collaboration avec les centres de confiance.

\section{Façonner implique aussi assumer des responsabilités!}

La valeur d'une banque de données dépend des informations qui l'alimentent. Cela signifie qu'il faut ras- sembler le plus grand nombre possible de données de bonne qualité afin de pouvoir réaliser des analyses représentatives et pertinentes.

Cet objectif ne pourra être atteint qu'avec des règles du jeu acceptées de tous et une répartition solidaire des droits et des devoirs. En d'autres termes, standards uniformes et normes de qualité acceptées de tous sont de mise lorsque les données sont réunies de manière décentralisée par différents «centres de collecte» (les centres de confiance), comme c'est le cas avec la banque de données. En effet, il faut absolument éviter que chacun établisse ses propres règles et normes car les données recueillies ne seraient plus compatibles avec la banque de données et elles perdraient une qualité essentielle: pouvoir être comparées entre elles.

\section{Conclusion}

NewIndex s'est fixé l'objectif de répondre au mandat du corps médical et d'agir en faveur des médecins en utilisant le puissant pool de données afin d'aller à la rencontre des enjeux à venir dans le domaine de la santé et de relever les défis en proposant des solutions réalistes.

Permettez-nous de conclure par de vifs remerciements à tous nos membres qui sont disposés depuis des années à transmettre leurs données et sans qui la banque de données n'existerait pas.

\section{Articles interactifs}

Vous souhaitez commenter cet article? II vous suffit pour cela d'utiliser la fonction «Ajouter un commentaire» dans la version en ligne. Vous pouvez également consulter les remarques de vos confrères sous: www.bullmed.ch/ numero-actuel/articles-interactifs/ 